\title{
Running on an unpredictable irregular surface changes lower limb biomechanics and subjective perception compared to running on a regular surface
}

\author{
Thorsten Sterzing ${ }^{{ }^{*}}$, Charlotte Apps ${ }^{1,2}$, Rui Ding ${ }^{1}$, Jason Tak-Man Cheung ${ }^{1}$ \\ From 4th Congress of the International Foot and Ankle Biomechanics (i-FAB) Community \\ Busan, Korea. 8-11 April 2014
}

\section{Background}

Irregular surface conditions, for instance, are present during trail running. Modified treadmills can be used to produce such surface conditions in a laboratory environment [1]. Gait variability on uneven shoe-surface interfaces is increased in walking $[2,3]$, hence the same may apply to running. This study examined the effects of an unpredictable irregular surface (UIS) on lower limb biomechanics, locomotion variability, and subjective perception during treadmill running.

\section{Methods}

Seventeen young, male, active participants ran at $8 \mathrm{~km} / \mathrm{h}$ on a treadmill with predictable regular surface (PRS) and with UIS. The UIS was created by randomly attaching EVA dome shaped inserts (b: $140 \mathrm{~mm}$ ) of different height $(10 \mathrm{~mm}$ and $15 \mathrm{~mm})$ and hardness (40 and 70 Asker C) to the treadmill. In-shoe plantar pressures $(200 \mathrm{~Hz}$, Pedar X System, Novel, Germany), lower limb kinematics (200 Hz, Vicon Peak, United Kingdom), and EMG signals of five lower limb muscles $(3000 \mathrm{~Hz}$, Telemyo $2400 \mathrm{G} 2$, Noraxon, USA) were recorded. Eight perception items were assessed subjectively (9-point Likert Scale). Biomechanical parameter mean magnitudes and mean standard deviations, as variability measure, of 16 steps were calculated. Variables were compared between surfaces by Wilcoxon signed rank tests $(\mathrm{p}<.05)$.

\footnotetext{
* Correspondence: thorsten@li-ning.com.cn

'Sports Science Research Center, Li Ning (China) Sports Goods Co Ltd, Beijing 101111, China

Full list of author information is available at the end of the article
}

\section{Results}

Step length decreased while step frequency increased on UIS ( $\mathrm{p}<.05)$. In-shoe pressure relative load magnitudes on UIS were increased at medial midfoot $(\mathrm{p}<.05)$, and decreased at lateral forefoot $(\mathrm{p}<.05)$. Relative load variability increased for all regions $(\mathrm{p}<.05)$. Runners had a flatter and less dorsiflexed foot strike (Table 1), alongside increased knee and hip flexion on UIS $(\mathrm{p}<.05)$. Whereas all sagittal joint angle magnitudes differed significantly, only knee and hip angles varied significantly more. Touchdown ankle inversion remained unchanged, whereas maximum eversion was significantly higher on UIS, and both were more variable $(\mathrm{p}<.05)$. Tibialis anterior and gastrocnemius medialis muscle activity magnitude and variability was similar, whereas peroneus longus activity was significantly increased, while not being more variable on UIS (Table 1). Subjectively, running on UIS was more challenging $(\mathrm{p}<.05)$.

\section{Conclusion}

Runners consciously applied a more alert kinematic lower limb posture at touchdown on UIS, with lower limb position more consistent for distal sagittal joint angles. Similar muscular activity of tibialis anterior and gastrocnemius medialis indicates that general muscle activity applied was sufficient to compensate the perturbation level in this study regarding sagittal plane ankle motion. Running on UIS increased gait variability, thus stimulating enhancement of motor control patterns, resembling a positive training mechanism [4]. 
Table 1 Magnitude (Mag) and variability (Var) of kinematic and EMG parameters, significant surface comparisons (PRS vs. UIS) indicated in bold.

\begin{tabular}{ccccccccccc}
\hline & \multicolumn{3}{c}{ Sagittal plane angles [deg] } & \multicolumn{5}{c}{ Normalized muscle activity during stance [\%] } \\
\cline { 2 - 12 } & \multicolumn{2}{c}{ Shoe to Surface } & \multicolumn{2}{c}{ Shoe to Shank } & \multicolumn{2}{c}{ Tibialis Anterior } & Gastrocnemius Med & Peroneus Longus \\
\hline & Mag & $\operatorname{Var}$ & Mag & Var & Mag & Var & Mag & Var & Mag & Var \\
\hline PRS & 20.8 & 2.1 & 9.7 & 1.3 & 24.4 & 3.3 & 42.1 & 5.6 & 42.7 & 5.8 \\
\hline UIS & 17.0 & 2.7 & 7.1 & 1.9 & 22.8 & 3.5 & 43.5 & 5.4 & 46.8 & 7.7 \\
\hline p-value & $\mathbf{. 0 0 1}$ & .102 & $\mathbf{. 0 0 1}$ & .055 & .149 & .492 & .831 & .586 & .025 \\
\hline
\end{tabular}

\section{Authors' details}

'Sports Science Research Center, Li Ning (China) Sports Goods Co Ltd, Beijing 101111, China. ${ }^{2}$ School of Sport and Exercise Sciences, Liverpool John Moores University, Liverpool, L3 3AF, UK.

Published: 8 April 2014

\section{References}

1. Voloshina AS, Kuo AD, Daley MA, Ferris DP: Biomechanics and energetics of walking on uneven terrain. J Exp Biol 2013, 216:3963-3970.

2. Gates DH, Wilken JM, Scott SJ, Sinitski EH, Dingwell JB: Kinematic strategies for walking across a destabilizing rock surface. Gait Posture 2012, 35:36-42.

3. Stögg| T, Müller E: Magnitude and variation in muscle activity during walking before and after a 10-week adaptation period using unstable (MBT) shoes. Footwear Sci 2012, 4(2):131-143.

4. Latash ML: The bliss of motor abundance. Exp Brain Res 2012, 217(1):1-5.

doi:10.1186/1757-1146-7-S1-A80

Cite this article as: Sterzing et al.: Running on an unpredictable irregular surface changes lower limb biomechanics and subjective perception compared to running on a regular surface. Journal of Foot and Ankle Research 2014 7(Suppl 1):A80.

Submit your next manuscript to BioMed Central and take full advantage of:

- Convenient online submission

- Thorough peer review

- No space constraints or color figure charges

- Immediate publication on acceptance

- Inclusion in PubMed, CAS, Scopus and Google Scholar

- Research which is freely available for redistribution

Submit your manuscript at www.biomedcentral.com/submit
C Biomed Central 